\title{
A conditional quantum phase gate between two 3-state atoms
}

\author{
X. X. Yi, X. H. Su, and L. You \\ School of Physics, Georgia Institute of Technology, Atlanta GA 30332, USA
}

(May 24, 2022)

We propose a scheme for conditional quantum logic between two 3-state atoms that share a quantum data-bus such as a single mode optical field in cavity QED systems, or a collective vibrational state of trapped ions. Making use of quantum interference, our scheme achieves successful conditional phase evolution without any real transitions of atomic internal states or populating the quantum data-bus. In addition, it only requires common addressing of the two atoms by external laser fields.

03.67.Lx, 32.80.Wr, 42.50.-p

Following the recent discovery of powerful applications for quantum computing algorithms [1,2], quantum information science [3] has witnessed significant progress and development on the experimental side. Among the variety of physical systems being explored for hardware implementations for quantum logic, atomic ion-traps and cavity QED systems are favored because of their demonstrated advantage when subjected to coherent manipulations.

Much experimental progress has been made with trapped ions, ranging from the deterministic creation of 4-ion internal state entanglement [4]; the generation of arbitrary superposition states of the quantized ion motion [5]; to the high fidelity quantum gate operations between two qubits [6]. While a high Q optical cavity with atomic qubits inside is an early candidate for quantum computation [7], it has been challenging to experimentally realize its full promise. In a recent paper [8], we delineated some of the technical difficulties and proposed an easier theoretical protocol for conditional phase dynamics between two 4-state atoms $[8,9]$. Let us recount the current difficulties with cavity QED base systems; 1) precisely localizing each atomic motional wave packet (to ensure the difficult Lamb-Dicke limit); 2) obtaining a double $\Lambda$-type, 6 -state, level diagram for each atom as required in the protocol of Ref. [7]; and 3) individually addressing each atom during the gate operation when both atoms are inside the cavity.

Recent developments in the synthesis of ion trap and cavity QED systems have allowed attainment of the Lamb-Dicke limit $[10,11]$. Such a composite system suggests possibilities for quantum communication of information stored in material atoms through cavity photons, in addition to processing quantum gates between several nearby atomic qubits.

In this paper, we present a new theoretical scheme for a quantum phase gate between two 3-state atoms. By making use of two-atom quantum interferences, our new protocol significantly reduces the effect of cavity decay and the atomic spontaneous emission during the gate operation. Furthermore, the external control of the system is made easier as the successful implementation of the phase gate involves no real atomic transitions or the presence of a cavity photon.

Our system consists of two 3-state atoms as in Ref. [12], each with two stable ground states $(|0\rangle$ and $|1\rangle)$ for storage of one qubit of quantum information, and an excited state $(|e\rangle)$ used for virtual transitions. In an experimental implementation, the $M_{F}=0$ clock transition states of alkali metal atoms are ideally candidates for the ground states because of their insensitivity to residual magnetic fields. Both atoms are assumed to couple (offresonantly) to an external laser field and the same single photon mode of the cavity as illustrated in Fig. 1 below. The conditional phase $(\phi)$ gate then is simply given by the following unitary evolution

$$
\begin{aligned}
|0\rangle_{A}|0\rangle_{B} & \rightarrow|0\rangle_{A}|0\rangle_{B} \\
|0\rangle_{A}|1\rangle_{B} & \rightarrow|0\rangle_{A}|1\rangle_{B} \\
|1\rangle_{A}|0\rangle_{B} & \rightarrow|1\rangle_{A}|0\rangle_{B} \\
|1\rangle_{A}|1\rangle_{B} & \rightarrow e^{i \phi}|1\rangle_{A}|1\rangle_{B},
\end{aligned}
$$

between the two atoms $\mathrm{A}$ and $\mathrm{B}$.

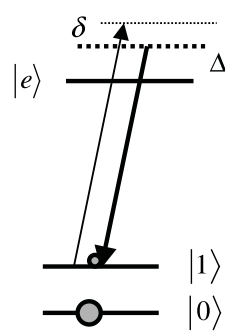

FIG. 1. A 3-state atom interacting with an external laser field and the cavity quantum field.

The effective model Hamiltonian of our system, including atomic spontaneous emission and cavity decays, is identical to that considered in Ref. [12] for a dynamical Stark phase gate between two trapped ions in a optical cavity.

$$
\begin{aligned}
H & =H_{A}+H_{B}+H_{C} \\
H_{\mu=A / B} & =\hbar\left(\omega_{e}-i \frac{\Gamma}{2}\right)|e\rangle_{\mu}\left\langle e\left|+\hbar \omega_{1}\right| 1\right\rangle_{\mu}\langle 1| \\
& +\frac{1}{2}\left[\Omega_{\mu}|e\rangle_{\mu}\left\langle 1\left|e^{-i \omega_{L} t}+g_{\mu}\right| e\right\rangle_{\mu}\langle 1| c+h . c .\right], \\
H_{C} & =\hbar\left(\omega_{C}-i \kappa\right) c^{\dagger} c
\end{aligned}
$$


where $H_{A / B}$ is the Hamiltonian for atom (A/B) interacting with the cavity mode and the external laser fields. $\Omega_{L}$ is the Rabi frequency of the external laser field $(|e\rangle \leftrightarrow|1\rangle)$ at frequency $\omega_{L}$ and $g_{\mu}$ is the single photon coherent coupling rate $(|e\rangle \leftrightarrow|1\rangle)$ of the cavity field. $\Gamma$ denotes the atomic spontaneous emission rate and $\kappa$ stands for the (one-side) cavity decay rate. We will not consider the position dependance of the cavity-atom coupling $g_{\mu}\left(\vec{r}_{\mu}\right)$, a good approximation in the Lamb-Dicke limit.

Our scheme works in the following limits: 1) both the external laser and the cavity field are strongly detuned, i.e., $\left|\Delta=\omega_{C}-\left(\omega_{e}-\omega_{1}\right)\right| \gg \Gamma, \kappa,\left|\Omega_{\mu}\right|,\left|g_{\mu}\right|$, and $|\delta| \sim$ 0 ; and 2) $\left|g_{\mu}\right|>\left|\Omega_{\mu}\right|$ and $\left|g_{\mu}\right|^{2} \gg \Gamma \kappa$ as required for the strong coupling. The dynamic Stark gate of Ref. [12] works when the classical field is resonant with the unperturbed atomic transition $|e\rangle \leftrightarrow|1\rangle$, i.e. $\omega_{L} \sim\left(\omega_{e}-\right.$ $\left.\omega_{1}\right)$, or $\delta=\omega_{L}-\omega_{C}=-\Delta$. Therefore, it is more sensitive to the atomic decay $\Gamma$. Our protocol, to be discussed below, works when both fields are strongly detuned and are resonant with each other, i.e. $\delta \sim 0$. It seems to work with reasonable losses due to both $\Gamma$ and $\kappa$.

In the following, we analyze in detail the two quantum interference transitions of the combined atom + cavity system that lead to the required conditional phase dynamics Eq. (1). We assume that the atom-laser and atom-cavity coupling strengths are adjustable so that $\Omega_{A}=-\Omega_{B}=\Omega$ and $g_{\mu}=g$. The latter condition can be easily established if we have the control over atomic positions inside the cavity, e.g. a linear atomic array intersecting the cavity at a right angle allows the placement of the two respective atoms in radially symmetric points of the (cylindrically symmetric) cavity. The former condition can be met when the plane wave laser field intersects from the side of the cavity such that the wave front delay between the two atoms (a distance $\vec{d}$ apart) corresponds to an odd number of $\pi$, i.e. $e^{i \vec{k}_{L} \cdot \vec{d}}=-1$.

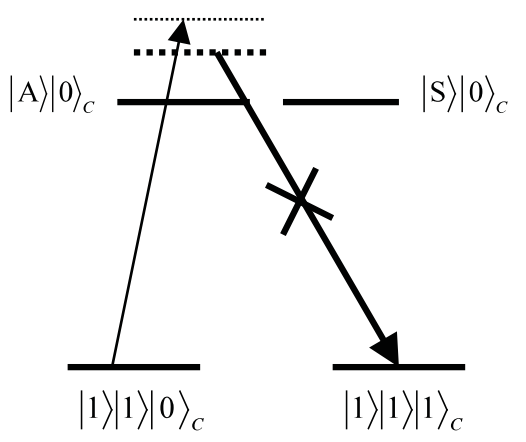

FIG. 2. The dominant transition path starting from state $|1\rangle_{A}|1\rangle_{B}|0\rangle_{C}$.

Within the above specific regime of parameters, the state $|0\rangle_{A}|0\rangle_{B}$ experiences no dynamics because the hyperfine splitting $\omega_{10}$ is much larger than $|\Delta|$ in all practical implementations. The state $|1\rangle_{A}|1\rangle_{B}$, on the other hand, accumulates a phase shift that increases linearly with time. As illustrated in Fig. 2, it couples first to the anti-symmetric one atom excited state $|\mathcal{A}\rangle=$ $\left(|e\rangle_{A}|1\rangle_{B}-|1\rangle_{A}|e\rangle_{B}\right) / \sqrt{2}$ because the choice of $\Omega_{\mu}$. The state $|\mathcal{A}\rangle$, however, CANNOT couple to the one cavity photon state $|1\rangle_{A}|1\rangle_{B}|1\rangle_{C}$ because of the choice $g_{\mu}=g$. In fact, state $|1\rangle_{A}|1\rangle_{B}|1\rangle_{C}$ is only coupled to the symmetric state $|\mathcal{S}\rangle=\left(|e\rangle_{A}|1\rangle_{B}+|1\rangle_{A}|e\rangle_{B}\right) / \sqrt{2}$. Further coupling to the two-atom excited state from $|\mathcal{A}\rangle$ is weakened because of the twice as large detuning. Thus the only dynamics for state $|1\rangle_{A}|1\rangle_{B}$ is a constant rate of its phase due to the AC Stark shift induced by the classical field $\Omega$, which we find to be $\phi(t)=-\frac{|\Omega|^{2}}{2(\Delta+\delta)} t$ when both decay rates are ignored.

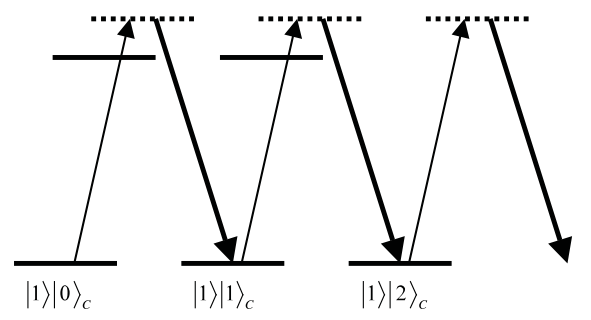

FIG. 3. The dominant single atom transition paths starting from atomic states $|1\rangle_{A}|0\rangle_{B}$ or $|0\rangle_{A}|1\rangle_{B}$ and an empty cavity.

Now let's look at states $|0\rangle_{A}|1\rangle_{B}$ and $|1\rangle_{A}|0\rangle_{B}$. It turns out that they accumulate no phase shifts at all as long as $\delta \sim 0$, again due to an interesting quantum interference. In this case the dominant transition paths are the single photon resonances between the classical and cavity fields as illustrated in Fig. 3. In the limit of $|g| \gg|\Omega|$, these resonant transitions become essentially summed by adopting the dressed state basis as in the standard Jaynes-Cummings model. All excited states then assume a doublet manifold given by

$$
\begin{aligned}
& |+, n\rangle=\sin \theta|1\rangle|n+1\rangle+\cos \theta|e\rangle|n\rangle, \\
& |-, n\rangle=\cos \theta|1\rangle|n+1\rangle-\sin \theta|e\rangle|n\rangle,
\end{aligned}
$$

with the corresponding eigenvalues

$$
E_{ \pm}(n)=-\frac{\Delta}{2} \pm \sqrt{\frac{\Delta^{2}}{4}+|g|^{2}(n+1)} .
$$

The mixing angle $\theta$ is determined from $\tan (2 \theta)=$ $-2 g \sqrt{n+1} / \Delta$. For the first excited state $(n=0)$, the above eigven-values also represent the detunings of states $| \pm, 0\rangle$ from the external laser field at $\omega_{L}=\omega_{C}$.

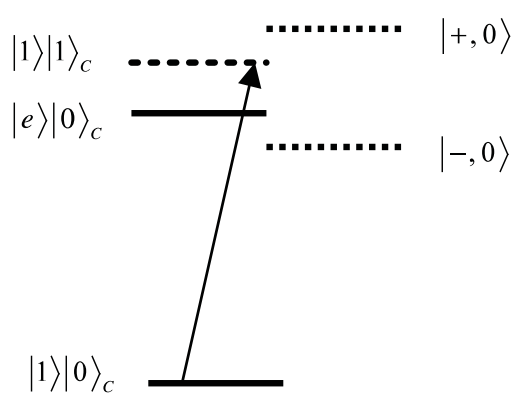


FIG. 4. The dominant single atom transition paths starting from atomic state $|1\rangle_{A}|0\rangle_{B}$ or $|0\rangle_{A}|1\rangle_{B}$ and an empty cavity, in the dressed state picture.

The weak classical field $\Omega$ then simply drives the atom from the ground state $|1\rangle_{\mu}|0\rangle_{C}$ to the first excited state doublet as in Fig. 4. Higher excited doublets are ignored again because of the large detuning. The AC Stark shift of the ground state $|1\rangle_{A}|0\rangle_{C}$ then simply results from the summation of the respective shifts of the doublet $| \pm, 0\rangle$. It is an easy exercise to show that the shift from the upper state $|+, 0\rangle$ (negatively detuned) exactly cancels that from the lower state $|-, 0\rangle$ (positively detuned), i.e.

$$
\delta_{10}=-\frac{|\Omega|^{2} \cos ^{2} \theta}{4 E_{+}(0)}-\frac{|\Omega|^{2} \sin ^{2} \theta}{4 E_{-}(0)}=0 .
$$

We have performed extensive numerical simulations with the full Hamiltonian Eq. (2). When both decays are ignored by taking $\Gamma=\kappa=0$, we find the above analytical insights to be completely accurate, i.e. we indeed execute the conditional phase gate Eq. (1). In fact, we find that the gate works with almost perfect (>99\% fidelity) even beyond the limit our interference based analysis implies, except that states $|1\rangle_{A}|0\rangle_{B}$ and $|0\rangle_{A}|1\rangle_{B}$ now also accumulate phases $\phi_{A}(t)$ and $\phi_{B}(t)$ respectively. The conditional phase then becomes $\phi(t)-\phi_{A}(t)-\phi_{B}(t)$. The phase gate of Ref. [12] involves a Rabi oscillation between state $|1\rangle_{A}|1\rangle_{B}$ and $|\mathcal{A}\rangle$. Thus it is less tolerant to residual phase shifts for states $|1\rangle_{A}|0\rangle_{B}$ or $|0\rangle_{A}|1\rangle_{B}$.

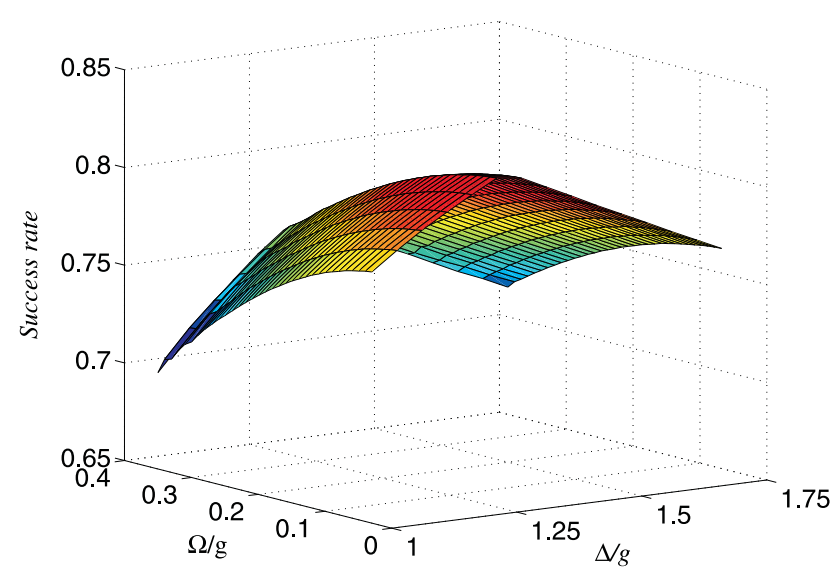

FIG. 5. The success rate for selected system parameters.

Figure 5 shows selected results for the dependence of the phase gate success rate on system parameters for $\Gamma=0.08|g|$ and $\kappa=0.05|g|\left(|g|^{2}=250 \Gamma \kappa\right)$. Quite promising results are obtained over a broad range of system parameters. The correspondingly fidelities are always approaching being perfect $(\mathcal{F} \geq 99.9 \%)$.

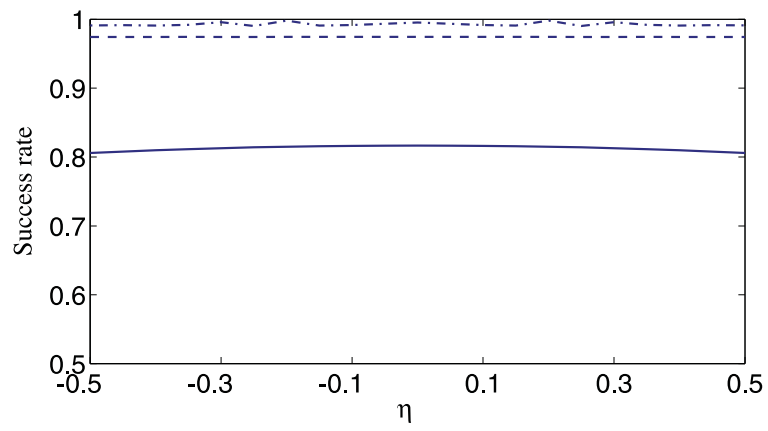

FIG. 6. The dependence of optimal success rate on $\eta$ for several choices of system parameters: $\Gamma=\kappa=0$ (dot-dashed), $\Gamma=0.05|g|, \quad \kappa=0$ (dashed line), and $\Gamma=0.08|g|, \kappa=0.05|g|$ (solid line).

In Fig. 6, we display the dependence of the optimal success rate on the relative phase $\eta$ between $\Omega_{A}=e^{i \eta} \Omega$ and $\Omega_{B}=-\Omega$. We see that the dependence is essentially flat. For $\eta \neq 0$, transitions from state $|1\rangle_{A}|1\rangle_{B}$ in Fig. 2 become modified in similar ways to those in Fig. 3 except the excited state is now a linear combination of $|\mathcal{A}\rangle$ and $|\mathcal{S}\rangle$. A similar reduction to doublet structures as in Fig. 4 occurs since $|g| \gg\left|\Omega_{\mu}\right|$. Thus the complete diagram is still essentially closed; now, however, the phase accumulation rate becomes smaller due to the cancellation of AC Stark shifts from the first excited doublet.

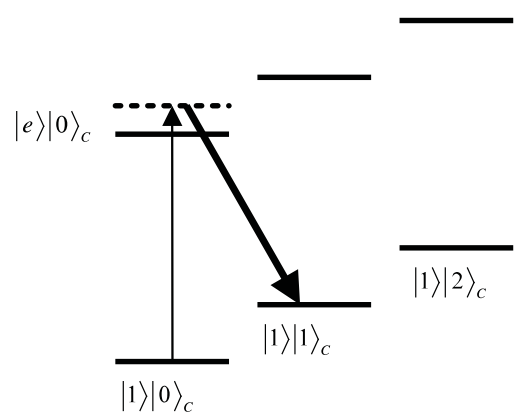

FIG. 7. An equivalent configuration of Fig. 1 for trapped ions. The internal state $|0\rangle$ and its associated vibrational band is not drawn as $\omega_{10} \gg \omega_{C}$.

Although the limit of $|g|^{2} \gg \Gamma \kappa$ is a challenging pursuit [13], it can nevertheless be expected to be reachable with optical cavity QED based systems soon $[14,15]$. On the other hand, for the state of art ion traps, $\kappa$ can be made exceedingly small and $\Gamma$ can be lowered using Raman transitions [4]. We now show that our model maps perfectly to an ion trap setup [16]. For the same type 3state atoms as illustrated in Fig. 1, the sideband of their collective vibrational mode is shared by the two atomic ions. We will use two external classical fields; one running wave drives the single atomic ion (off-resonantly) at the carrier frequency $|1\rangle_{\mu}|n\rangle_{C} \leftrightarrow|e\rangle_{\mu}|n\rangle$ (insensitive to the motion); and the other drives the red motional sideband as illustrated in Fig. 7. The equivalent cavity coupling $g_{\mu}$ then becomes in this case $R_{\mu}$, the Rabi frequency for 
the second laser (much stronger than the first one at the carrier frequency) times its respective Lamb-Dicke parameters of the harmonic vibration for each of the atoms $\eta_{\mu}$ [17]. The Hamiltonian of the system is then again described by Eq. (2), except now the index ' $\mathrm{C}$ ' refers to the collective vibrational state and $c^{\dagger}(c)$ denotes the creation (annihilation) of a vibration quanta. We will require $|\Delta| \ll \omega_{C}$ to prevent any internal excited state with a non-zero vibrational quanta from participating the gate dynamics.

If the collective center of mass vibrational mode is used, then $\eta_{\mu}=\eta$, the same as in the cavity QED model. We could also use the two ion breathing mode, then $\eta_{A}=-\eta_{B}$, which leads to $g_{A}=-g_{B}=g$. If we then take $\Omega_{A}=\Omega_{B}=\Omega$ instead, the same theory as developed above applies.

In Fig. 8, we show selected results of the success rate for $\kappa=0$, a situation close to an ion trap implementation.

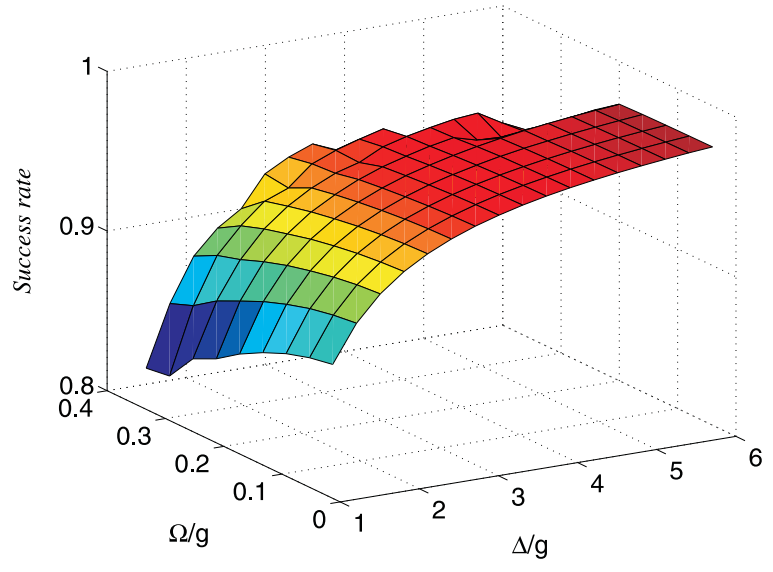

FIG. 8. Same as in Fig. 5 but for $\Gamma=0.05|g|$ and $\kappa=0$.

In conclusion, we have discovered a new protocol for performing a quantum phase gate between two atomic qubits. We have explained its operating mechanism in terms of quantum interference effects. In addition, our protocol can also be explained in terms of a quantum Zeno subspace [18] or an environment induced decoherence free space as in other cavity QED based theories $[12,19]$. We believe this new protocol is more advantageous when compared to other known cavity QED protocols. It requires only common addressing of the two atoms during the gate operation; it uses 3 internal states for each atomic qubit, and applies to both cavity QED and trapped ion systems; Furthermore, its successful implementation involves no real transitions of atomic states or populating the cavity or the collective motional state quantum data-bus. In the ideal operation limit, this protocol thus becomes reasonably insensitive to motional dephasing or heating because the Stark phase due to an external plane wave laser is position independent.

We thank Dr. M. Plenio for helpful communications. This work is supported by a grant from NSA, ARDA, and
DARPA under ARO Contract No. DAAD19-01-1-0667, and by a grant from the NSF PHY-0113831.

[1] P. W. Shor, Proc. 35th Ann. Symp. on the Foundations of computer science, Ed. S. Goldwasser, (Los Alamitos, CA IEEE computer society).

[2] L. K. Grover, Phys. Rev. Lett. 79, 325(1997).

[3] D. Deutsch, Proc. R. Soc. A 400, 97(1985); D. Deutsch, Proc. R. Soc. A 425, 73(1989).

[4] C. A. Sackett et al., Nature(London) 404, 256(2000).

[5] A. Ben-Kish, B. DeMarco, V. Meyer, M. Rowe, J. Britton, W.M. Itano, B.M. Jelenković, C. Langer, D. Leibfried, T. Rosenband, and D.J. Wineland, (quantph/0208181).

[6] B. DeMarco, A. Ben-Kish, D. Leibfried, V. Meyer, M. Rowe, B.M. Jelenković, W.M. Itano, J. Britton, C. Langer, T. Rosenband, and D.J. Wineland, (quantph/0208180).

[7] T. Pellizzari, S. Gardiner, J. I. Cirac, and P. Zoller, Phys. Rev. Lett. 75, 3788(1995).

[8] L. You, X. X. Yi, and X. H. Su, (quant-ph/0209096).

[9] J. Pachos and H. Walther, (quant-ph/0111088).

[10] G. R. Guthohrlein, M. Keller, K. Hayasaka, W. Lange, and H. Walther, Nature 414, 49(2001).

[11] A. B. Mundt, A. Kreuter, C. Becher, D. Leibfried, J. Eschner, F. Schmidt-Kaler, and R. Blatt, Phys. Rev. Lett. 89, 103001 (2002).

[12] E. Jane, M. B. Plenio, and D. Jonathan, Phys. Rev. A 65, 050302 (2002).

[13] P. Bertet, S. Osnaghi, P. Milman, A. Auffeves, P. Maioli, M. Brune, J. M. Raimond, and S. Haroche, Phys. Rev. Lett. 88, 143601 (2002).

[14] C. J. Hood et al., Science 287, 1447 (2000); A. C. Doherty, T. W. Lynn, C. J. Hood, and H. J. Kimble, Phys. Rev. A 63, 013401 (2001).

[15] P. W. H. Pinkse et al., Nature 404, 365 (2000); M. Hennrich et al., Phys. Rev. Lett. 85, 4872 (2000).

[16] S. Schneider, D. F. V. James, and G. J. Millburn, J. Mod. Opt. 47, 499 (2000).

[17] Almut Beige, (quant-ph/0205070).

[18] P. Facchi and S. Pascazio, Phys. Rev. Lett. 89, 080401 (2002).

[19] A. Beige, D. Braun, B. Tregenna, and P. L. Knight, Phys. Rev. Lett. 85, 1762 (2000). 